among SLE patients. Further investigation is needed to fully elucidate associations between biological (unpredictable of SLE symptoms), social (informational and appraisal sources of social support), and community level (public awareness campaigns) factors influencing disease. Findings also point to the necessity of integrating community organizations, physicians, and friends and family of patients with SLE into capacity building interventions aimed at improving HRQOL.

Funding Source(s): Summer Undergraduate Research Award (Washington University in St, Louis)

\section{FREQUENCY OF HYDROXYCHLOROQUINE RETINOPATHY IN THE HOPKINS LUPUS COHORT}

${ }^{1}$ Michelle Petri ${ }^{*},{ }^{2}$ Marwa Elkhalifa, ${ }^{1}$ Jessica Li. 'Johns Hopkins University School of Medicine; ${ }^{2}$ Johns Hopkins University

\subsection{6/lupus-2019-Ism.15}

Background The Kaiser-Permanente study projected hydroxychloroquine (HCQ) retinopathy rates of $40 \%$ after 20 years of use. We have prospectively followed SLE patients in the Hopkins lupus cohort to compare.

Methods Patients in the Hopkins cohort are seen quarterly for assessment of disease activity and lupus complications. Yearly ophthalmology examinations are requested. Patients, if insurance allows, are referred to the Wilmer Retina group. Four tests are performed: OCT, ERG, MP-1 and FAF.

Results Patients had a variety of retinal testing done, with optical coherence testing most frequent. Table 1 shows the concordance of a test abnormality with the retina expert opinion.

The concordance of the test abnormality with the retina expert opinion showed the following sensitivity and specificity, respectively: OCT 93\%, 84\%; ERG 100\%, 51\%; MP1 100\%, 70\%; and FAF 83\%, 76\%.

The frequency of retinopathy increased with years of HCQ use [number of retinopathies per total patients (percent frequency)]: 5 years or less, 1/103 (0.97\%); 6-10 years, 2/109

\begin{tabular}{|c|c|c|}
\hline Test & Retinopathy N (\%) & No Retinopathy N (\%) \\
\hline \multicolumn{3}{|c|}{ Ocular Coherence Tomography (OCT) } \\
\hline Abnormal & $25(93 \%)$ & $133(16 \%)$ \\
\hline Normal & $2(7 \%)$ & $721(84 \%)$ \\
\hline Total & 27 & 854 \\
\hline \multicolumn{3}{|c|}{ Electroretinogram (ERG) } \\
\hline Abnormal & $15(100 \%)$ & $195(49 \%)$ \\
\hline Normal & $0(0 \%)$ & $201(51 \%)$ \\
\hline Total & 15 & 396 \\
\hline \multicolumn{3}{|c|}{ Microperimetry (MP1) } \\
\hline Abnormal & $17(100 \%)$ & $144(30 \%)$ \\
\hline Normal & $0(0 \%)$ & $331(70 \%)$ \\
\hline Total & 17 & 475 \\
\hline \multicolumn{3}{|c|}{ Fundal Autoflourescence (FAF) } \\
\hline Abnormal & $19(83 \%)$ & $165(24 \%)$ \\
\hline Normal & $4(17 \%)$ & $536(76 \%)$ \\
\hline Total & 23 & 701 \\
\hline
\end{tabular}

(1.83\%); 11-15 years, 3/91 (3.30\%); 16-20 years, $11 / 96$ (11.46\%); and 21 or more years, 6/75 (8.00\%).

Conclusions In agreement with the American Academy of Ophthalmology, OCT appears to be the optimum test for yearly monitoring. The frequency of retinopathy was much lower in our prospective study than estimated by the KaiserPermanente study. Our data also show the need for ophthalmologists with retinopathy expertise to interpret retina testing, as screening tests are frequently abnormal due to causes other than HCQ retinopathy. Stopping HCQ based on an abnormal test without confirmation from a retinopathy expert could needlessly deprive an SLE patient of an important medication.

Funding Source(s): The Hopkins Lupus Cohort was funded by NIH Grant R01-AR069572.

\section{HYDROXYCHLOROQUINE BLOOD LEVELS PREDICT RETINOPATHY IN SLE}

${ }^{1}$ Michelle Petri* ${ }^{*}{ }^{1}$ Jessica Li, ${ }^{2}$ Marwa Elkhalifa, ${ }^{1}$ Daniel Goldman. ${ }^{1}$ Johns Hopkins University School of Medicine; ${ }^{2} J o h n s$ Hopkins University

10.1136/lupus-2019-Ism.16

Background Hydroxychloroquine (HCQ) retinopathy after 10 years or more of use is more frequent than previously appreciated. This led to new ophthalmology guidelines that

\begin{tabular}{|c|c|c|c|c|}
\hline & No n (\%) & Yes $\mathbf{n}(\%)$ & $\mathrm{p}$ & P for Trend \\
\hline \multicolumn{5}{|l|}{ Characteristic } \\
\hline Sex & & & 0.1029 & \\
\hline Female & $475(96.2)$ & $19(3.8)$ & & \\
\hline Male & $39(90.7)$ & $4(9.3)$ & & \\
\hline Ethnicity & & & 0.3804 & \\
\hline White & $238(94.4)$ & $14(5.6)$ & & \\
\hline Black & $215(96.4)$ & $8(3.6)$ & & \\
\hline Other & $61(98.4)$ & $1(1.6)$ & & \\
\hline Age & & & $<0.0001$ & $<0.0001$ \\
\hline$<45$ & $215(99.5)$ & $1(0.5)$ & & \\
\hline $45-59$ & $175(95.6)$ & $8(4.4)$ & & \\
\hline $60+$ & $124(89.9)$ & $14(10.1)$ & & \\
\hline HCQ max & & & 0.0340 & 0.0143 \\
\hline 1 (0 to 1182$)$ & $161(98.8)$ & $2(1.2)$ & & \\
\hline 2 (1183 to 1752$)$ & $157(95.2)$ & $8(4.8)$ & & \\
\hline 3 (1753 to 6281$)$ & $153(93.3)$ & $11(6.7)$ & & \\
\hline HCQ duration & & & 0.0006 & 0.0002 \\
\hline 1 ( 0 to 8 years) & $158(98.8)$ & $2(1.2)$ & & \\
\hline 2 (9 to 15 years) & $139(97.2)$ & $4(2.8)$ & & \\
\hline 3 (16 to 48 years) & 154 (90.1) & $17(9.9)$ & & \\
\hline BMI & & & 0.1701 & 0.0160 \\
\hline$<20$ & $50(98.0)$ & $1(2.0)$ & & \\
\hline $20-25$ & $171(97.7)$ & $4(2.3)$ & & \\
\hline $25-30$ & $159(95.2)$ & $8(4.8)$ & & \\
\hline $30-35$ & $76(95.0)$ & $4(5.0)$ & & \\
\hline $35+$ & $58(90.6)$ & $6(9.4)$ & & \\
\hline HTN Ever & & & 0.0020 & \\
\hline Yes & $276(93.2)$ & $20(6.8)$ & & \\
\hline No & $238(98.8)$ & $3(1.2)$ & & \\
\hline
\end{tabular}


changed the recommended dosing from $6.5 \mathrm{mg} / \mathrm{kg}$ to $5 \mathrm{mg} /$ $\mathrm{kg}$. However, it is not clear that the lower dose of hydroxychloroquine will have the same efficacy for SLE activity or the same protective role against cardiovascular risk factors and thrombosis. We asked whether hydroxychloroquine blood levels could help identify those at greater future risk of retinopathy.

Methods We analyzed data on 537 SLE patients from a clinical cohort who had repeated assessments of HCQ blood concentrations, and were evaluated one or more times for retinopathy (300 single retinopathy exam, 149 two and 88 three or more assessments). The patients were 92\% female and 42\% Caucasian. Hydroxychloroquine blood levels were performed as previously described. In our analysis, HCQ toxicity was defined dichotomously by a retina expert: all those with a value of No or Possible were categorized as not having HCQ toxicity, and those who had a Yes were categorized as having it. Mean and maximum HCQ blood concentration over all cohort visits prior to the final retinopathy assessment were calculated. Risk of HCQ toxicity was then assessed in tertiles defined by these variables.

Results Significant risk factors for retinal toxicity are shown in table 1.

Conclusions Our data show that the risk of HCQ retinopathy is higher in men and Caucasians. As expected, it is higher in older patients and with greater duration. We also found that BMI and hypertension were predictive of HCQ retinopathy. For the first time, our data show the utility of HCQ blood levels in predicting retinopathy. This would allow clinicians to either decrease dose or increase monitoring in those with high blood levels.

Funding Source(s): The Hopkins Lupus Cohort was funded by NIH Grant R01-AR069572.

\section{TYPE I INTERFERON MODULATES ADAM17 ACTIVITY IN PHOTOSENSITIVE LUPUS MOUSE MODELS}

${ }^{1}$ Thomas M Li*, ${ }^{1}$ Noa Shwartz, ${ }^{1}$ William D Shipman, ${ }^{2}$ Theresa T Lu. ${ }^{1}$ Hospital for Special Surgery; ${ }^{2}$ Autoimmunity and Inflammation Program and Pediatric Rheumatology, Hospital for Special Surgery

\subsection{6/lupus-2019-Ism.17}

Background Systemic lupus erythematosus (SLE) is an autoimmune disease characterized by systemic disease flares, renal complications, photosensitivity, and other clinical manifestations. A research area of particular interest to our lab is photosensitivity, an immune system reaction precipitated by ultraviolet radiation (UVR) exposure that results in epidermal keratinocyte apoptosis and contributes to overall skin inflammation. Importantly, many SLE patients attribute a decrease in quality of life due to photosensitivity. Our lab has recently identified a mechanism in which a disintegrin and metalloprotease 17 (ADAM17) located on Langerhans cells (LCs) limits UVR-induced keratinocyte apoptosis and skin injury via keratinocyte epidermal growth factor receptor (EGFR) stimulation in mice and humans. While we have delineated the downstream effects of UVR-induced ADAM17 activity and expression in healthy and SLE skin, factors that regulate ADAM17 remain poorly understood. Previous studies have shown that elevated levels of type I interferon (IFN) are characteristic of SLE patients. Here, we hypothesize that type I interferon may regulate ADAM17 in LCs in lupus models.

Methods To quantify ADAM17 activity, we developed an assay that uses flow cytometry and co-culture systems. We use this assay in in vitro models and in vivo mouse models. To quantify the relative ratio of IFN-inducible genes, we use quantitative polymerase chain reaction (qPCR). To apply IFN-k to mice, we solubilize the protein and paint it on murine back skin.

Results In vitro methods showed that type I IFN was sufficient to reduce LC ADAM17 activity, and in vivo models showed that type I IFN receptor blockade corrected the LC ADAM17 defect in photosensitive lupus mouse models. We are analyzing qPCR data.

Conclusions We show that type I interferon (IFN) can reduce ADAM17 activity on epidermal cells. We also show that type I interferon receptor blockade in lupus mouse models rescues ADAM17 activity. Our data together suggest that type I IFN in lupus may contribute to Langerhans cell dysfunction and propensity to photosensitivity in SLE.

Funding Source(s): None

\section{AN UNCOMMON OVERLAP OF TWO COMMON RHEUMATOLOGICAL DISORDERS}

Sandesh Guleria*, Ankur Jindal, Sanjeev Naganur, Deepti Suri, Surjit Singh. Postgraduate Institute of Medical Education and Research, Chandigarh, India

\subsection{6/lupus-2019-Ism.18}

Background Juvenile systemic lupus erythematosus (SLE) is a heterogeneous multisystem autoimmune disease. Kawasaki disease $(\mathrm{KD})$ is a common vasculitic disorder in children that manifests with fever and mucocutaneous involvement. While overlap of childhood SLE with other rheumatologic disorders has been described, it is extremely unusual in the
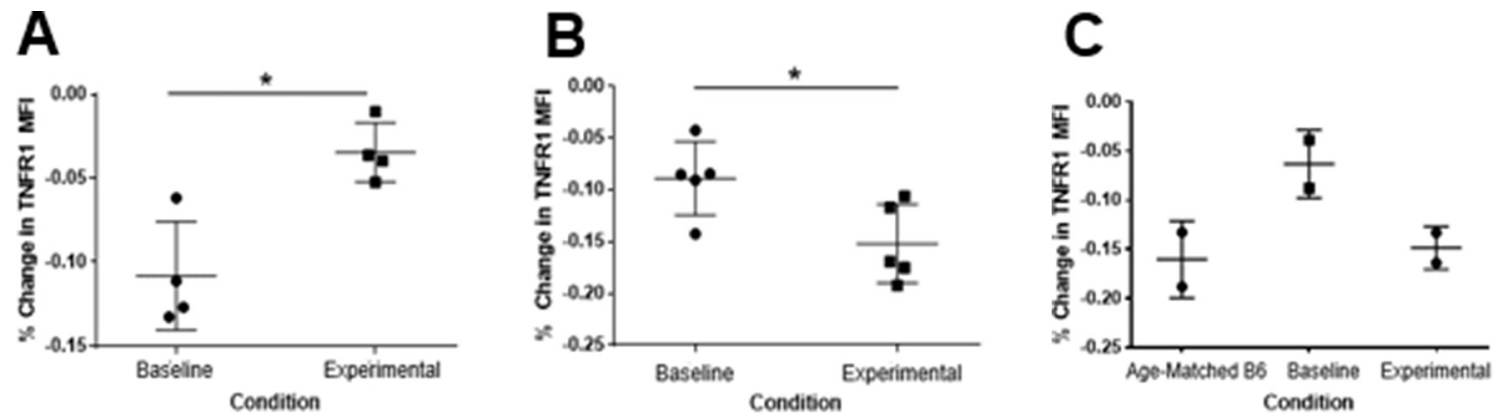

Abstract 17 Figure 1 Interferon is a sufficient and necessary condition to downregulate ADAM17 activity 